\title{
Vitiligo na criança e doença da tireóide*
}

\author{
Childhood vitiligo and thyroid disease
}

\author{
Nurimar Conceição Fernandes ${ }^{1}$
}

\author{
Márcia Maria Cajueiro Campos ${ }^{2}$
}

\begin{abstract}
Resumo: A associação entre vitiligo e tireoidopatia na criança é discutível. Cinquenta crianças com vitiligo e $40 \mathrm{sem}$ vitiligo foram submetidas às dosagens séricas de anticorpos antitireoide e hormônio tireoestimulante. Um caso (grupo teste) e um caso controle mostraram títulos de TSH acima do limite normal; o vitiligo não representou maior risco para tireoidopatia.

Palavras-chave: Anticorpos; Criança; Glândula tireoide; Tireotropina; Vitiligo
\end{abstract}

O vitiligo é observado na tireoidopatia autoimune: tireoidite linfocítica crônica (doença de Hashimoto), causa mais comum do hipotireoidismo; doença de Graves, etiologia mais comum do hipertireoidismo. Os dados de frequência na criança com vitiligo são conflitantes ${ }^{1-6}$ A doença de Hashimoto se caracteriza por bócio nodular difuso indolor e elevação de antitireoglobulina e enzima tireoperoxidase (TPO). O hipertireoidismo é raro na criança e acomete mais as meninas com história familiar de tireoidopatia; há poliúria, nictúria, exoftalmia, tireóide aumentada, tremor fino de mãos (que se apresentam quentes e úmidas) e língua, taquicardia, PA divergente, emagrecimento e dificuldade de concentração. $\mathrm{O}$ diagnóstico é confirmado por elevação de $\mathrm{T}_{3}$ e $\mathrm{T}_{4}$ livres e totais. Para triagem laboratorial de disfunção tireoidiana, propõe-se a dosagem de tireotropina (TSH) que identifica doença não suspeitada ou oligossintomática. A positividade do anticorpo anti-TPO ocorre nas tireoidopatias autoimunes. ${ }^{7,8}$
Desenho do estudo: caso controle em que os fatores de exposição calculados são as alterações séricas dos níveis de TSH e presença de autoanticorpos.

Cinqüenta crianças (0-12 anos) com vitiligo (grupo teste), selecionadas por sorteio randomizado entre 120 portadoras da doença e 40 (0-12 anos) não portadoras recrutadas no momento de coleta de sangue para exames de rotina por outras patologias, foram avaliadas de 30/01/2003 a 30/01/2006 no IPPMG-UFRJ.

Critérios de exclusão (casos e controles): uso de imunossupressores, anticonvulsivantes, salicilatos, iodeto, sulfas, isoniazida, penicilina; diabetes mellitus; anemias perniciosa/hemolítica; síndromes de Down/Turner; doença de Addison; colagenoses; nefropatia; hepatopatia; cardiopatia e desnutrição.

$\mathrm{O}$ vitiligo foi definido clinicamente por máculas branco-marfim, de borda bem delimitada e hiperpigmentada com poliose.

O exame para disfunção da tireóide incluiu pal-

Aprovado pelo Conselho Consultivo e aceito para publicação em 1.11.08

* Trabalho realizado no Instituto de Puericultura e Pediatria Martagão Gesteira (IPPMG) e Hospital Universitário Clementino Fraga Filho da Universidade Federal do Rio de Janeiro (HUCFF/UFRJ) - Rio de Janeiro (RJ), Brasil.

Conflito de interesse: Nenhum / Conflict of interest: None

Suporte financeiro: Curso de Pós-Graduação em Dermatologia - Faculdade de Medicina da Universidade Federal do Rio de Janeiro (UFRJ).

Professora associada, Faculdade de Medicina, Universidade Federal do Rio de Janeiro. Instituto de Puericultura e Pediatria Martagão Gesteira. Hospital Universitário Clementino Fraga Filho - Rio de Janeiro (RJ), Brasil.

2 Curso de Pós-Graduação em Medicina (área de concentração: Dermatologia), Faculdade de Medicina, Universidade Federal do Rio de Janeiro - Rio de Janeiro (RJ), Brasil. 
pação da glândula, frequência cardíaca, pressão arterial e observação de sudorese, poliúria, nictúria, tremor fino das mãos e língua.

As amostras de sangue venoso colhidas pela manhã (jejum de oito horas) foram mantidas congeladas a $-70^{\circ} \mathrm{C}$ até que todas fossem coletadas para dosagens hormonal e imunológica simultâneas.

$\mathrm{O}$ método de quimioluminescência Diagnostic Products Corporation (DPC) - foi utilizado para dosagens séricas de TSH $(\mathrm{N}=0,4-4 \mu \mathrm{U} / \mathrm{mL})$ e anti-TPO (Negativo: < 35UI/mL; Positivo: > $35 \mathrm{UI} / \mathrm{mL})^{7}$

Termo de consentimento assinado pelos responsáveis foi obtido após esclarecimento.

No grupo teste ( 9 meninos brancos / 8 não brancos e 18 meninas brancas / 15 não brancas) foram observados: 24 casos da forma generalizada, 9 da segmentar, 7 da focal, 5 da mucosa e 5 da acrofacial. O grupo controle incluiu 9 meninos brancos / 11 não brancos e 13 meninas brancas / 7 não brancas; 17 (34\%) crianças no grupo teste e 8 (20\%) controles relataram história familiar de tireoidopatia; a proporção foi a mesma nos dois grupos $(\mathrm{p}=0,064)$ (Tabela 1$)$.

Nível sérico elevado de TSH $(6,56 \mu \mathrm{U} / \mathrm{ml})$ em um caso do grupo teste (10 anos, não branca, forma mucosa vulvar) e em um controle: 7 anos, branca, com psoríase, história familiar positiva para tireoidopatia $(4,27 \mu \mathrm{U} / \mathrm{ml})$. O anti-TPO foi negativo em ambos os grupos (Tabela 2).

Não houve evidência de doença tireoidiana em nenhum dos dois grupos.

As publicações disponíveis são séries de casos nos quais se incluem adultos, adolescentes e crianças; são vagas em relação aos exames laboratoriais utilizados, o que dificulta a correta avaliação da associação vitiligo/tireoidopatia na faixa pediátrica. ${ }^{1,2,4,9,10}$

Apenas três estudos observacionais com crianças ( $\leq 12$ anos) portadoras de vitiligo foram publicados: um caso de tireoidopatia/625 (0,16\%); ${ }^{5}$ um caso de hipotireoidismo/73 $(1,3 \%) ;^{3}$ dois casos com anticorpos antitireoidianos $/ 59(3,4 \%) ;^{3}$ um caso com alteração de TSH/66 (1,6\%). ${ }^{3}$

Especulam-se o significado do anti-TPO na ausência de sinais clínicos e a importância de screening nos doentes de vitiligo. Os resultados neste estudo de caso controle demonstraram que não houve maior risco para tireoidopatia e formação de anticorpos para tireoide. No estágio atual de conhecimento, mesmo se detectada e tratada alguma disfunção da glândula, nenhuma modificação do vitiligo é observada.

TABELA 1 : Distribuição dos grupos segundo dados de história familiar de tireoidopatia

\begin{tabular}{llllll}
\hline \multirow{2}{*}{ Grupos } & \multicolumn{2}{l}{ Com história familiar } & \multicolumn{2}{l}{ Sem história familiar } & Totais \\
\cline { 2 - 6 } & $\mathbf{N}$. & $\%$ & $\mathbf{N}$. & $\%$ & \\
Teste & 17 & $34 \%$ & 33 & $66 \%$ & 50 \\
Controle & 8 & $20 \%$ & 32 & $80 \%$ & 40 \\
\hline Totais & 25 & 65 & & 90 \\
\hline Fonte: & IPPMG/UFRJ (2003-2006) & & &
\end{tabular}

TABeLA 2 : Distribuição dos títulos séricos de TSH e anti-TPO

\begin{tabular}{llll}
\hline Grupos & N. de casos & TSH $~$ & Anti-TPO + \\
\cline { 2 - 4 } & & N. & N. \\
Teste & 50 & 1 & - \\
Controle & 40 & 1 & - \\
\hline
\end{tabular}

Fonte: IPPMG/UFRJ (2003-2006)

Convenção:

- nenhum caso

$>$ aumentada 


\section{REFERÊNCIAS}

1. Pagovich OE, Silverberg JI, Freilich E, Silverberg NB. Thyroid abnormalities in pediatric patients with vitiligo in New York city. Cutis. 2008;81:463-6.

2. Iacovelli P, Sinagra JLM, Vidolin AP, Marenda S, Capitanio B, Leone G. Relevance of thyroiditis and of other autoimmune diseases in children with vitiligo. Dermatology. 2005;210:26-30.

3. Silva CMR, Pereira LB, Gontijo B, Ribeiro GB. Vitiligo na infância: características clínicas e epidemiológicas. An Bras Dermatol. 2007;82:47-51.

4. Kurtev A. Thyroid function and autoimmunity in children and adolescents with vitiligo. J Eur Acad Dermatol Venereol. 2004;18:99-117.

5. Handa S, Dogra S. Epidemiology of childhood vitiligo: a study of 625 patients from North Índia. Pediatric Dermatol. 2003;20:207-10.

6. Kuhl IC, Weissbluth ML, Bakos L, Wollmann TM. Pesquisa de autoanticorpos e função tireoidiana em pacientes portadores de vitiligo e alopecia areata. An Bras Dermatol. 1995;70:421-5.

7. Buescu A, Grego Filho J. Propedêutica nas doenças da tireoide. In: Carneiro AJV, Fraga EG, Pimentel ML, Vargas SSM, editores. Clínica médica. Doenças da tireóide. São Paulo: Atheneu; 2003. p.1-23.
8. Guimarães MM. Doenças tireoidianas na infância. In: Carneiro AJV, Fraga EG, Pimentel ML, Vargas SSM editores. Clínica médica. Doenças da tireóide. São Paulo: Atheneu; 2003. p.119-44.

9. Kakourou T, Kanaka-Gantenbein C, Papadopoulou A. Increased prevalence of chronic autoimmune (Hashimoto's) thyroiditis in children and adolescents with vitiligo. J Am Acad Dermatol. 2005;53:220-3.

10. Schallreuter KE, Lemke R, Brandt O. Vitiligo and other diseases: coexistence or true association ? Study on 321 patients. Dermatology. 1994;188:269-75.

ENDEREÇO PARA CORRESPONDÊNCIA / MAILING ADDRESS: Nurimar C. Fernandes

Rua Alexandre de Gusmão, 28 - Ap. 201

20520120 Rio de Janeiro RJ

Tel./fax: 21 2568-4158

E-mail: nurimarfernandes@terra.com.br

Como citar este artigo/How to cite this article: Fernandes NC, Campos MMC. Vitiligo na criança e doença da tireóide. An Bras Dermatol. 2009;84(2):200-2. 